\title{
Grèce antique et Grèce moderne
}

\section{Samuel Baud-Bovy}

\section{(2) OpenEdition \\ Journals}

Édition électronique

URL : http://journals.openedition.org/ethnomusicologie/2298

ISSN : 2235-7688

Éditeur

ADEM - Ateliers d'ethnomusicologie

Édition imprimée

Date de publication : 1 janvier 1988

Pagination : 77-86

ISBN : 2-8257-0159-9

ISSN : 1662-372X

Référence électronique

Samuel Baud-Bovy, «Grèce antique et Grèce moderne », Cahiers d'ethnomusicologie [En ligne], 1| 1988,

mis en ligne le 15 août 2011, consulté le 30 avril 2019. URL : http://journals.openedition.org/

ethnomusicologie/2298 


\title{
GRÈCE ANTIQUE ET GRÈCE MODERNE*
}

\author{
Samuel Baud-Bovy
}

Alors que les savants grecs du siècle dernier, que ce soit pour faire plaisir aux philhellènes ou pour combattre la théorie de Fallermayer sur la slavisation de la Grèce au Moyen Age, mettaient tout en œuvre pour prouver que les Grecs modernes étaient les descendants directs des Grecs anciens, les générations suivantes se lassèrent de ce culte exclusif voué aux ancêtres. Il arrivait même à Palamas, le poète national du $\mathrm{XX}^{\mathrm{e}}$ siècle naissant, de s'insurger contre cette mentalité :

Les tombes, même augustes, sont des tombes :

Nous voulons, nous aussi, une place au soleil.

Mais aujourd'hui, où l'existence d'une civilisation grecque se suffisant à ellemême est reconnue dans tous les domaines, nous n'avons plus la moindre raison de ne point admettre que certains éléments du monde antique sont restés vivaces dans la Grèce moderne.

Je vous en citerai deux exemples : une danse, le syrtós, et un vers, le décapentasyllabe iambique.

Le syrtós que j'ai à l'esprit n'est ni le syrtós crétois, appelé aussi khaniótikos du nom de la ville de La Canée - cela ne m'étonnerait pas qu'il remonte à l'époque où la population de cette ville, chef-lieu de l'administration ottomane, était en majorité turque -, ni le syrtós de Constantinople ou des îles de l'Archipel, ni bien entendu le "syrtaki» (improvisé par Anthony Quinn dans le film de Zorba); c'est le syrtós de la Grèce continentale, connu sous le nom de kalamatianós.

Il serait faux d'inférer de cette appellation que la danse en question est originaire de la ville de Calamata en Messénie. Les musiciens populaires, pour la distinguer des autres syrtós, l'appelaient ainsi parce que les vers qu'ils chantaient en la jouant parlaient de «mouchoirs de Calamata». A Chypre, par exemple, les paroles du kalamatianós que publie Georges Averof dans son ouvrage Danses populaires chypriotes (1978: 103-104) étaient ou bien :

Si tu vas à Calamata, si tu reviens, Dieu voulant, rapporte-m'en z-un mouchoir à nouer autour du cou. 
ou bien :
Un mouchoir de Calamata
autour de ton cou de neige,
tu le portes, tu rayonnes
comme l'astre du matin.

Le kalamatianós est dansé de nos jours dans toute la Grèce, mais il est propre à la Grèce continentale. L'ethnomusicologue W. Swets, qui a étudié spécialement les danses de la péninsule balkanique et leurs rythmes, arrive à la conclusion que le rythme grec par excellence est celui du kalamatianós (1958: 394). De son côté, Thrasybule Georgiades, dans son livre intitulé Der griechische Rhythmus, note que le kalamatianós est la danse cyclique la plus répandue en Grèce continentale (1949: 99).

Le rythme du kalamatianós est habituellement identifié au mètre que les anciens appelaient l'«épitrite». Dans la langue des mathématiciens, les દ̇лıópı désignent des fractions dont le numérateur est supérieur d'une

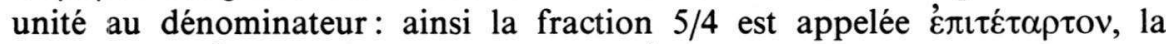

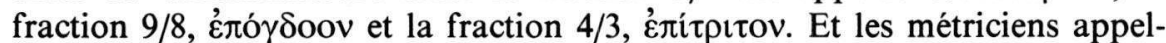
lent "pied épitrite" le mètre dont l'un des temps compte quatre unités de durée $(\mu o ́ p l \alpha)$, et l'autre trois. Ces sept unités de durée correspondent à trois syllabes longues et à une syllabe brève. Selon la place occupée par la brève, on distinguait l'épitrite premier $\left.\int\right\rfloor \vdots \downarrow$, l'épitrite second $J \int \vdots J$, l'épitrite troisième $J \int \vdots d$ et l'épitrite quatrième

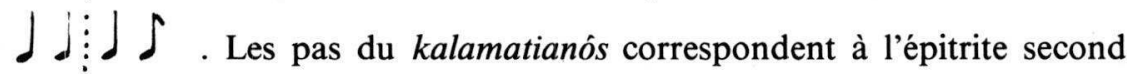
(plus rarement à l'épitrite premier), puisque son premier pas correspond à trois petites unités de temps, trois croches diraient les musiciens ( $D_{J}=J_{\text {. }}$ ), alors que les deux suivants, plus légers, durent chacun deux unités $(\nabla J=J J$ ).

Pour le constater, il suffit qu'un Grec du continent entende jouer trois mesures de kalamatianós: inconsciemment, tout son être est aussitôt saisi par le rythme fondamental des pas de la danse (Exemple $N^{\circ} 1$ ).
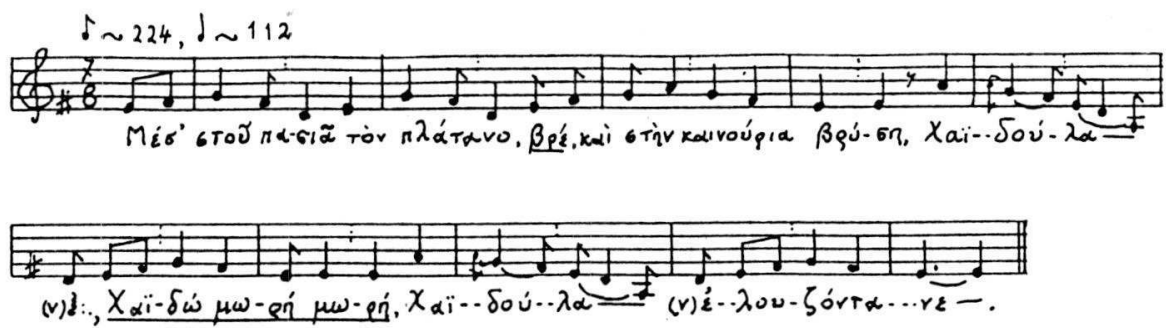

Exemple $\mathrm{N}^{\circ} 1$ : Kalamatianós du village de Khirades dans le district de Mégalopolis (Arcadie). Chant: Anna Maniati-Noni. Enregistrement: Simon Karas et Maria Voura. Disque 33 t. SDNM 113, Chansons du Péloponnèse, face I 3. 
Dans l'ouvrage cité, Georgiades a exposé les raisons pour lesquelles il avait cessé d'admettre le rapprochement du kalamatianós avec l'épitrite antique. Il compare la place occupée par le kalamatianós dans le folklore de la Grèce moderne à la place qu'occupait le vers dactylique homérique dans les danses cycliques des anciens (1949: 110). Invoquant les exemples de vers dactyliques cités par Denys d'Halicarnasse, il en conclut que, sous les pas des danseurs, la syllabe longue du dactyle était abrégée et que sa durée ne valait pas deux brèves, mais une brève et demie. Le rapport de la longue à la brève devenait donc $1,5 / 2$, soit $3 / 4$, disait Georgiades. Mais il n'avait pas remarqué que dans les deux cas cités par Denys, la longue s'abrège pour que la déclamation suggère le mouvement rapide, accéléré, décrit par le poète: l'impétuosité du vent qui pousse le vaisseau d'Ulysse (Odyssée IX 39) et le rocher de Sisyphe qui, à peine hissé au sommet de la pente, dégringole à nouveau (Odyssée XI 598).

La rapidité est telle que, d'après Aristoxène, le vers devient "irrationnel ", c'est-à-dire que la plus petite unité de temps (le «temps premier», $\chi \rho o ́ v o \varsigma$ $\pi \rho \tilde{\omega} \tau \mathrm{\varsigma})$ n'est plus perceptible, elle qui est indispensable pour qui veut mesurer les durées. Or, dans le kalamatianós cité plus haut, l'unité de durée est la croche $\left(~ \int\right.$ ), dont la durée est parfaitement perceptible, puisqu'elle suffit à l'articulation de certaines syllabes du vers :

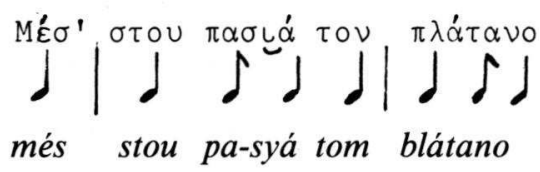

Un autre argument de Georgiades contre l'identification du rythme du kalamatianós avec l'épitrite (1949: 101) est que l'épitrite est un pied rare dans la poésie antique et que, toujours selon Aristoxène, il ne se prête pas à une composition suivie, qu'en d'autres termes il ne peut être répété et ne se rencontre que mêlé à d'autres mètres. C'est la raison pour laquelle aucun traité de métrique antique ne mentionne de vers épitritiques; il n'est question que de "dactylo-épitrites", c'est-à-dire de mètres qui combinent des dactyles et des épitrites.

Un vers comme celui-ci, pris dans la Paix d'Aristophane (v. 301) ${ }^{1}$ :

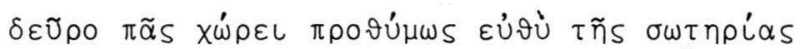

$$
\begin{aligned}
& |-v-1-v--|-v--|-v-\wedge|
\end{aligned}
$$

est classé parmi les vers trochaïques; il est qualifié de «tétramètre trochaïque catalectique", du fait que son dernier mètre n'a que trois syllabes au lieu de quatre. Dans une certaine mesure, l'analyse est justifiée: dans un mètre trochaïque qui groupe, en principe, deux trochées $(-\cup-u$, soit 6 ل 8 ل $\vdots\rfloor \int$ ), le second trochée peut être remplacé par un spondée, 
formé de deux longues (--); mais dans ce cas, pour ne pas rompre la cadence $\mathrm{du}$ vers et pour que les mètres ne cessent d'être isochrones, les longues du

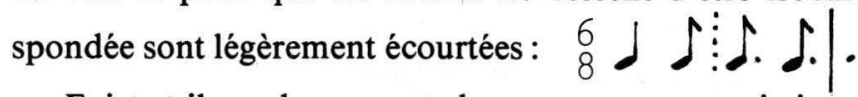

Existe-t-il, au demeurant, des vers que nous puissions à bon droit qualifier d'épitritiques, en raison de la régularité avec laquelle ils enchaînent les épitrites? Il faut répondre par l'affirmative.

L'un d'eux, en tout cas, a été lu par Pausanias (VI 14, 9-10) à Olympie, sur un monument funéraire représentant un homme qui tenait deux flûtes:

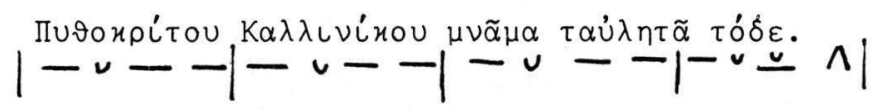

Ceci est le tombeau de Pythocritos le flûtiste, fils de Callinicos.

Ce Pythocritos était un musicien célèbre originaire de Sicyone qui, au $\mathrm{VI}^{\mathrm{e}}$ siècle avant notre ère, avait remporté six fois le prix du concours de flûte de Delphes et jouait son instrument à Olympie pour encourager les athlètes dans la palestre, tout comme aujourd'hui les "Anastenarides" dansant pieds nus sur le feu, soutenus par la clarinette et le tambour.

Au témoignage de Dion Chrysotome (Orationes II 33), c'est dans le même mètre qu'était rédigée l'interdiction qu'Alexandre le Grand fit afficher sur la maison natale de Pindare, lorsqu'il incendia Thèbes:

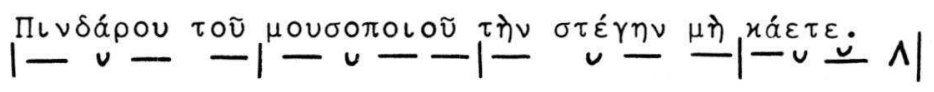

De Pindare, le poète lyrique, ne brûlez point la demeure !

Nous savons par ses biographes que Pindare, comme tous les Thébains de haute naissance, jouait de la flûte ; même le nom de son maître est conservé. Il ne peut donc être fortuit si, la première fois où il composa en trimètres épitritiques, ce fut dans l'unique ode qu'il dédia à un vainqueur du concours de flûte aux jeux pythiques, Midas d'Agrigente. Chacune des quatre strophes de la $\mathrm{XII}^{\mathrm{e}}$ Pythique s'achève en effet par un trimètre épitritique :

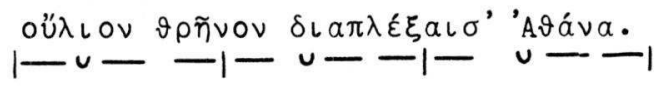

Nous avons vu qu'Aristoxène rangeait l'épitrite parmi les mètres "qui ne se prêtent pas à une composition suivie»; cependant, nous trouvons dans les odes de Pindare, et plus tard de Simonide et de Bacchylide, des séquences de trois, quatre, cinq épitrites.

Ainsi, chaque strophe de la $\mathrm{IX}^{\mathrm{e}}$ Pythique, écrite à la gloire de héros thébains, s'achève par un tétramètre épitritique sur le modèle :

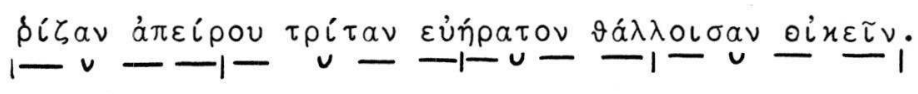


De même, la III ${ }^{\mathrm{e}}$ Isthmique, en l'honneur du Thébain Mélissos, commence par un tétramètre épitritique :

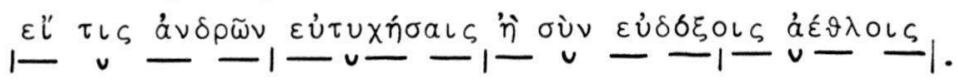

et finit solennellement par un pentamètre épitritique catalectique :

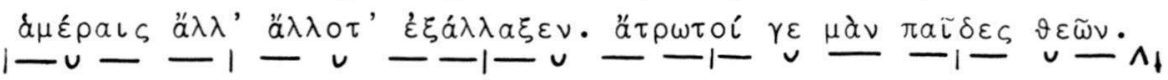

Quant au vers d'Aristophane cité plus haut, dont on aura noté la régularité épitritique, il est loin d'être isolé. Il ouvre la párodos (entrée du chœur) de la Paix, et les choreutes qui le chantent figurent les Grecs épris de paix que le vigneron Trygée a invités à délivrer la Paix du gouffre où l'a jetée Polémos, le dieu de la guerre. Le rythme dansant de leur entrée a été préparé par l'appel de Trygée, composé en vers iambiques. On sait que dans l'iambe, la brève précède la longue : $\cup-$. Dans le mètre iambique, constitué de deux iambes, la première brève peut être remplacée par une longue : - - $\cup-$. Dans ce cas, comme nous l'avons vu pour le mètre trochaïque, les deux longues successives sont légèrement abrégées: $\quad 6 \delta .5 \vdots\rfloor J$, ou alors le mètre se change en épitrite troisième: $\downarrow \downarrow \vdots \vdots \delta$. Tel est le mètre fondamental de tous les vers de l'appel de Trygée; mais il faut noter aussi que son premier vers est emprunté à une chanson populaire à la mode, la "chanson de Datis". Si nous le lisons en élevant la voix sur les syllabes accentuées:

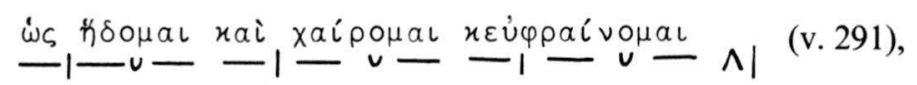

nous entendons non pas des épitrites troisièmes, mais des épitrites seconds précédés d'une levée. Leur correspondance est complète avec le kalamatianós de l'Exemple $\mathrm{N}^{\circ} 1$ :

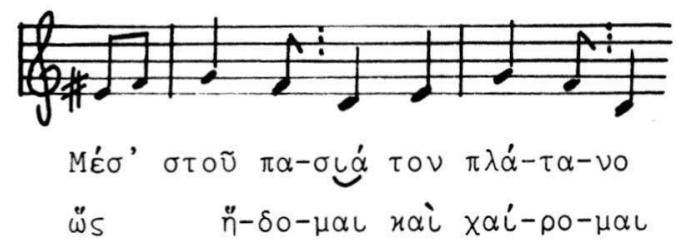

Tous les vers suivants dans l'appel de Trygée sont taillées sur le modèle de l'incipit de la chanson de Datis :

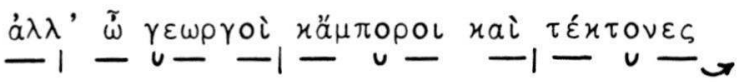

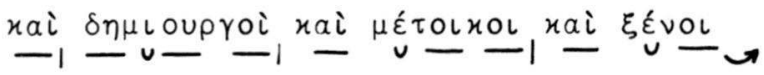

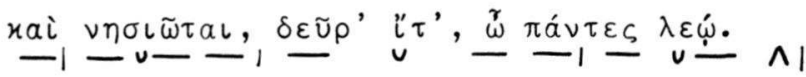


De tout ce que nous avons vu, comment ne pas conclure que des vers épitritiques se rencontraient dans la poésie populaire des anciens, et notamment dans leurs chansons à danser? Cela éclaire aussi le sens de deux vers de Pindare, au début de la $\mathrm{III}^{\mathrm{e}}$ Olympique, qui ont embarrassé les philologues. En voici la traduction :

C'est ainsi que la Muse m'assista lorsque je découvris une manière inédite et brillante d'adapter

à la sandale dorienne le chant qui embellit les fêtes.

Par «sandale dorienne", Pindare n'entend évidemment pas le mode dorien, l'échelle dorienne, comme l'ont voulu certains exégètes, mais, comme l'a judicieusement noté un commentateur ancien, le "rythme dorien», on dirait mieux : le pas dorien ("sandale», $\pi \varepsilon \delta i ́ \lambda \varphi$ étant le substitut d'un prosaïque "pied», $\pi 0 \delta i)$. Et ce "pas dorien», ce devait être précisément l'épitrite, puisque les vers en question combinent dactyles et épitrites:

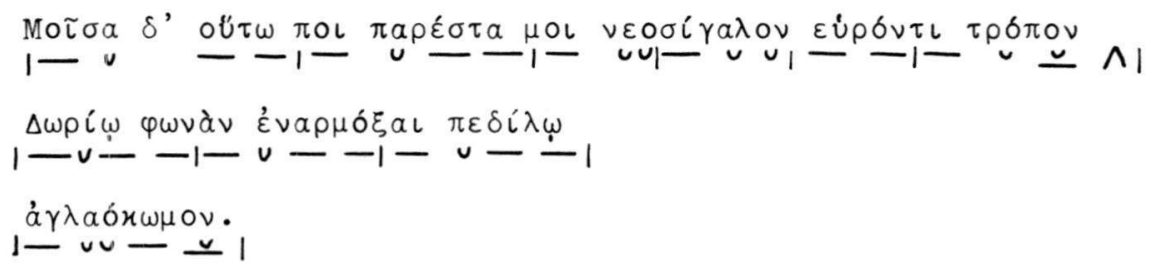

Ce n'est pas un hasard si Pindare, dans cette ode triomphale, exalte deux héros particulièrement vénérés chez les Doriens, à savoir les Dioscures.

Résumons les résultats de notre enquête :

Primo: Il existait chez les anciens des chansons populaires dont le rythme était épitritique. Nous en voulons pour preuve la "chanson de Datis" conservée dans la Paix d'Aristophane; le fait que Datis dise $\chi \alpha i \rho o \mu \alpha \iota$ au lieu du pur attique $\chi \alpha i p \omega$ montre bien qu'il est un homme du peuple.

Secundo: Les flûtistes de Grèce continentale devaient jouer, de préférence, des airs sur le rythme de l'épitrite. Nous en voulons pour preuve l'inscription gravée sur le monument de Pythocritos.

Tertio: Ce rythme a été introduit par le Thébain Pindare dans l'ode triomphale. Et enfin: c'est sur le rythme épitritique que devaient danser les Doriens.

Il me reste à rappeler qu'à Karditsa en Béotie fut trouvée une inscription du premier siècle de notre ère, gravée par les habitants du bourg d'Akraiphié en l'honneur de leur compatriote Epaminondas (I.G. VII 2712). Parmi les bienfaits du personnage, l'inscription mentionne qu'il «fit célébrer avec magnificence les processions traditionnelles et exécuter religieusement la danse ancestrale des syrtós".

Dès l'Antiquité, les habitants de la Grèce continentale ont donc dansé le syrtós que nous appelons kalamatianós, dont on a raison d'identifier le rythme avec l'épitrite. 
Venons-en maintenant au second exemple de continuité entre la Grèce antique et la Grèce moderne: le vers de quinze syllabes. Je ne puis traiter ce sujet sans évoquer le grand savant, l'ami très cher que nous avons perdu, Linos Politis. Sa dernière communication faite devant ses collègues de l'Académie d'Athènes avait pour titre: Vues nouvelles sur l'origine et la structure du décapentasyllabe (1981 : 211-228). Son intention première était de résumer les acquisitions récentes de la recherche des néohellénistes concernant la provenance de ce vers, devenu le vers populaire grec par excellence, celui qui, pour reprendre les termes de Séféris cités par Politis, «a approché, plus que tout autre rythme, l'ondoiement profond de notre parler ».

Politis terminait la revue de ces études en s'arrêtant à l'article publié par Michael Jeffreys dans les Dumbarton Oaks Papers (1974). Jeffreys y affirme que le vers qualifié de "politique» (c'est-à-dire de "vulgaire») par les érudits byzantins provient du versus quadratus latin :

Salva Roma, salva patria, salvus est Germanicus.

Cette théorie fort improbable (qui néglige le fait que le versus quadratus est de rythme trochaïque, alors que le vers "politique» est de rythme iambique) reposait sur la prétendue constatation qu'un écart de plus de mille ans séparait le dernier tétramètre iambique, dans une comédie de Ménandre, du premier vers politique écrit au début du $\mathrm{X}^{\mathrm{e}}$ siècle, et que par conséquent toute filiation était exclue.

Arrivé à ce point, L. Politis fut obligé de modifier son exposé, du fait qu'un collègue l'avait informé de la publication récente, par un papyrologue anglais, d'un texte du $\mathrm{II}^{\mathrm{e}}$ siècle après Jésus-Christ contenant des vers dont la structure était semblable à celle du décapentasyllabe (Haslam 1981). "Les vers du papyrus, écrit Politis, sont des tétramètres iambiques catalectiques, vers connus de la métrique antique, utilisés surtout dans la comédie [...]. L'éditeur estime que des vers de ce type devaient être assez populaires à la fin de l'Antiquité et se rencontrer d'ordinaire dans les genres littéraires inférieurs. 'J'ose proposer (ajoute l'éditeur) que le vers politique byzantin, encore fort usité de nos jours, tire son origine directement et, serait-on tenté de dire, par quelques voies encore inexplorées, du vers dont notre papyrus offre un spécimen caractéristique.' Bien entendu, poursuit Politis, les vers sont prosodiques (c'est-àdire fondés sur l'alternance des syllabes longues et brèves) et non pas toniques (c'est-à-dire fondés sur l'accent des mots); il est remarquable cependant que tous, sans exception, sont accentués sur l'avant-dernière syllabe, et portent donc le principal signe distinctif du décapentasyllabe moderne. On en jugera par un exemple:

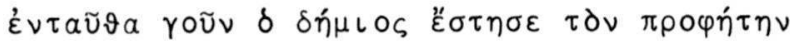

La trouvaille, conclut Politis, apporte un témoignage inattendu, exceptionnellement précoce, et pose un problème à la recherche philologique $»$.

Ajoutons que Haslam compare en note les vers qu'il publie avec les vers d'un autre papyrus, contenant une collection de textes magiques, incantations, exorcismes et sorts. Wessely $(1885: 31)$, le premier éditeur du papyrus en 
question, avait distingué, à côté d'invocations à différentes divinités, écrites en hexamètres dactyliques et en trimètres iambiques, une invocation à la Lune qui est effectivement en tétramètres iambiques catalectiques. Bien que le papyrus soit daté du $\mathrm{IV}^{\mathrm{e}}$ siècle, il semble reproduire des modèles sensiblement antérieurs. Dans ses tétramètres iambiques, on n'observe point encore la tendance à accentuer la pénultième, comme dans le papyrus révélé par Haslam. Voici le début du papyrus magique :

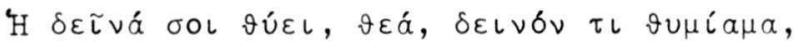

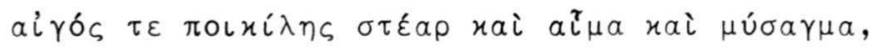

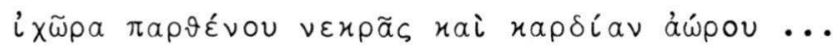

On peut dire que certains vers d'Aristophane étaient déjà plus proches du vers politique, comme ce vers du Plutus qui fait l'effet d'une variante de la chanson de Datis citée plus haut:

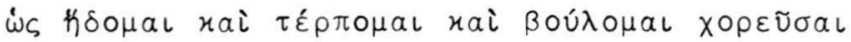

$$
\begin{aligned}
& \begin{array}{lllllllllllllll}
1 & \underline{2} & 3 & 4 & 5 & \underline{6} & 7 & 8 & 9 & \underline{10} & 11 & 12 & 13 & \underline{14} & 15
\end{array}
\end{aligned}
$$

On remarque à l'audition que les accents de durée coïncident avec les accents de hauteur, comme pour mieux suggérer l'envie irrépressible qu'éprouve le coryphée de danser.

De tels tétramètres iambiques ne se trouvent que chez les comiques; les tragiques devaient les considérer comme inappropriés à l'art élevé, comme "vulgaires", annonçant en cela le préjugé des érudits byzantins contre le vers "politique».

C'est la raison qui me fait considérer comme populaire un distique auquel ni moi-même, ni ceux qui ont étudié l'origine du vers politique, n'avions prêté attention. Il s'agit encore d'une invocation, non pas à la Lune, comme dans le papyrus magique, mais à la Muse ; elle est connue depuis 1581, date à laquelle elle a été publiée par Vincentio Galilei, le père de l'astronome (1602: 96). Il l'avait découverte dans un manuscrit du XVe siècle (alors qu'elle existe déjà dans des manuscrits du XIII ${ }^{e}$ et du $\mathrm{XIV}^{\mathrm{e}}$ ), parmi des poèmes mis en musique par Mésomède, compositeur crétois de la cour d'Hadrien, er parmi des traités de théorie musicale :

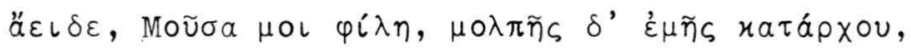

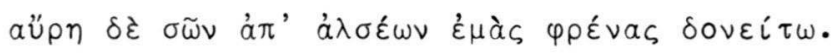

Chante, ma Muse bien-aimée, daigne entonner ma mélopée, et qu'un souffle venu de tes bosquets sacrés

fasse vibrer mon âme !

Alors que les poèmes de Mésomède sont écrits en dialecte dorien archaïsant et sur des mètres antiques, le distique ci-dessus, d'une langue simple, est composé dans le vers populaire qu'est le tétramètre iambique cata- 
lectique. Il est vrai que ses accents musicaux ne coïncident pas toujours avec des longues; ils sont rendus cependant par la mélodie. Cette mélodie nous est conservée dans la notation antique, que nous lisons sans peine grâce aux tables des théoriciens.

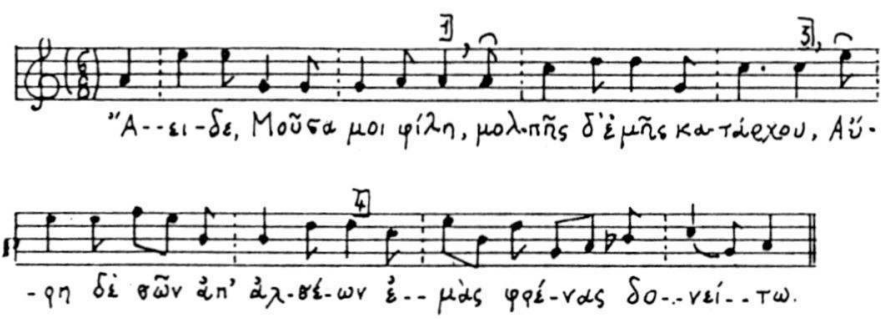

Exemple $\mathrm{N}^{\circ} 2$ : Invocation à la Muse. Voir note 2.

La première fois que je chantai cette mélodie (Exemple $\mathrm{N}^{\circ} 2$ ) d'après la transcription de Théodore Reinach ${ }^{2}$, elle me rappela aussitôt un air que j'avais enregistré en Crète en 1954, lors de la mission des Archives musicales de folklore de $\mathbf{M}^{\text {me }}$ Melpo Merlier (Exemple $\mathrm{N}^{\mathrm{o}} 3$ ). Les vers que chantait le vieux Vardas sont tirés du premier chant du poème versifié d'Erotocritos, du XVII ${ }^{\mathrm{e}}$ siècle (vers 1629-1640, édition St. Alexiou 1980). On constate qu'ils ressemblent étroitement à l'invocation à la Muse, par le rythme, par l'ambitus presque identique, par les degrés où aboutissent les quatre hémistiches.

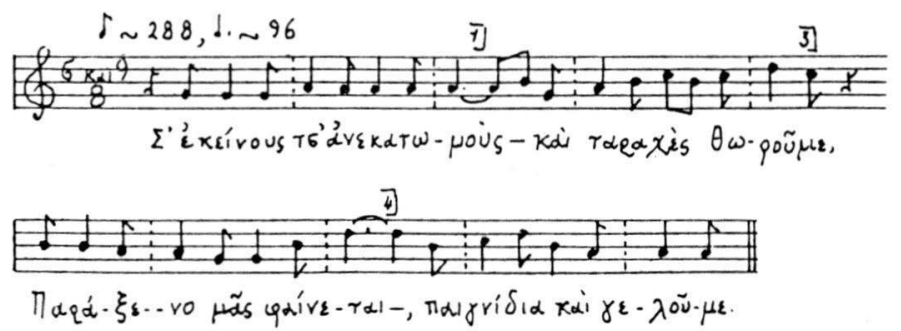

Exemple $\mathrm{N}^{\circ} 3$ : Extrait d' Erotocritos, chant I, vers 1629-1630. Chant : Georges E. Vardas, dit Kazanis, du village de Krousta, district de Merabello (Crète orientale). Enregistrement: Archives musicales de folklore, Athènes, bande P I v 1.

Je pense en conclusion que pour approcher la musique antique, restée si mystérieuse à tant d'égards, la meilleure manière est d'étudier la chanson populaire authentique, et ce non pas dans l'esprit du savant Coray, qui condescendait à lire Erotocritos et d'autres "monstres" de la Muse populaire, comme on cajole la "laide servante" pour obtenir les bonnes grâces de la "sublime maîtresse", mais comme un vieillard qui se laisse émouvoir par une belle jeune fille dont le visage lui rappelle la grand-mêre qu'il avait aimée dans sa jeunesse. 


\section{Notes}

* Le 15 mai 1984, sur invitation du professeur P. D. Mastrodimitris, directeur du Département de philologie grecque moderne, Samuel Baud-Bovy faisait dans l'amphithéâtre Spyridion Lambros de l'Université d'Athènes une conférence destinée aux étudiants de la

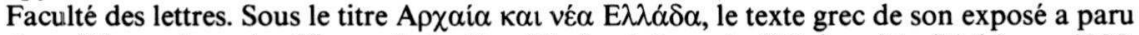
dans l'Annuaire scientifique de la Faculté des lettres de l'Université d'Athènes, 1985, p. 549-560; il s'agit du dernier article imprimé de son vivant par le savant genevois. La version française qu'on va lire est due à Bertrand Bouvier.

1 J'utilise les signes de la métrique : - pour la syllabe longue, dont la durée correspond à la noire ( $\int$ ), $\cup$ pour la syllabe brève, dont la durée correspond à la croche $\left(\int\right)$, et $\wedge$ pour le soupir ( $\boldsymbol{Z}$ ).

2 Non pas celle qu'on lit dans son ouvrage La musique grecque (1926:194), mais celle qu'il publia dans l'article intitulé "L'hymne à la Muse" (1896:3). Je ne m'arrêterai point ici aux menues retouches que j'apporte à la transcription de Reinach.

\section{Bibliographie}

\section{AVEROF Georges}

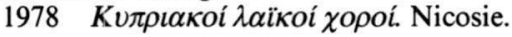

GALILEI Vicentio

1602 Dialogo della Musica antica e moderna. Florence. ( $2^{e}$ éd.).

GEORGIADES Thrasyboulos

1949 Der griechische Rhythmus. Hambourg.

HASLAM M.W.

1981 "Narrative about Tynouphis in Prosimetrum». In: Papyri Greek and Egyptian. London. P.35-45 (ed. in honour of Eric Gardner Turner).

\section{JEFFREYS Michael}

1974 "The Nature and Origins of the Political Verse». In : Dumbarton Oaks Papers 28: 142-195.

\section{POLITIS Linos}

1981 "Vues nouvelles sur l'origine et la structure du décapentasyllabe» (en grec).

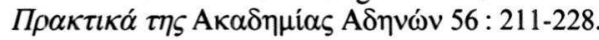

REINACH Théodore

1896 "L'hymne à la Muse». Revue des études grecques IX : 1-22.

1926 La musique grecque. Paris.

SWETS Wouter

1958 «Development of unusual metrical types in the folk music of the Balkans and Asia Minor ». Antiquity and Survival II : 387-404.

\section{WESSELY Carl}

1885 "Griechische Zauberpapyri von Paris und London". Denkschriften der kais. Akademie der Wissenschaften, Philol.-hist. Klasse, 36, 2. Abt., Vienne, p. 27-298. 